\title{
Servicios bibliotecarios para inmigrantes venezolanos en Medellín
}

\author{
Johnny Alejandro Ceballos Ospina \\ Universidad de Antioquia, Medellín, Colombia / \\ alej0861028@gmail.com / https://orcid.org/oooo-0oo2-8884-5643
}

\section{Resumen}

En los últimos años, el fenómeno migratorio venezolano es una realidad que se ha hecho presente en todo el territorio colombiano y, por ende, en la ciudad de Medellín. Es importante hacer hincapié en el rol de la biblioteca pública y su capacidad de responder a estos nuevos escenarios por medio de la adaptación de servicios que permitan proporcionar a los inmigrantes, información y herramientas necesarias para integrarse con éxito en la cultura local y regional. Este artículo pretende ofrecer un panorama general sobre el constante flujo de ciudadanos venezolanos en las bibliotecas públicas de la ciudad y, finalmente, se proponen algunas estrategias de servicios, programas y actividades encaminadas a mejorar las condiciones de vida de estos nuevos usuarios.

\section{Abstract}

Library services for venezuelan immigrants in Medellín. In recent years, the Venezuelan migratory phenomenon is a reality that has been present in the Colombian territory and, therefore, in Medellin city. It is important to emphasize the role of the public library and its ability to respond to these new scenarios through the adaptation of services that provide immigrants with information and tools to integrate

\section{Palabras clave}

Biblioteca pública Venezolanos Inmigrantes Estrategias themselves successfully into the local and regional culture. This paper aims to provide an overview of the constant flow of Venezuelan citizens in the public libraries of the city and, finally, some service strategies, programs and activities are proposed, in order to improve the living conditions of these new users.

Keywords

Public library Venezuelans Immigrants Strategies 


\section{Introducción}

La crisis política, económica y social que actualmente está viviendo el hermano país de Venezuela, ha hecho que miles de ciudadanos venezolanos emprendan un éxodo migratorio a otros países en busca de nuevas oportunidades que les permitan mejorar la calidad de vida y la de sus familias. Muchas de estas personas han elegido Colombia, ya sea como país transitorio o para establecerse de manera permanente, teniendo en cuenta que un número considerable, lo hicieron por medio del Permiso Especial de Permanencia (PEP) que se creó para regularizar la situación migratoria de quienes ingresaron de forma legal al territorio colombiano, sumando los nacionales del vecino país que cuentan con cédula de extranjería y que actualmente residen en ciudades con mayor cantidad poblacional como Medellín (Migración Colombia, 2018c). Además, están quienes ingresaron de manera irregular a través de los siguientes pasos fronterizos:

» Colombia-Venezuela: Paso de frontera Puente Internacional Francisco de Paula Santander (Cúcuta).

»Colombia-Venezuela: Paso de frontera Internacional Simón Bolívar (Cúcuta).

»Colombia-Venezuela: Paso de frontera Puente Internacional La Unión (Cúcuta).

»Colombia-Venezuela: Paso de frontera Puente Internacional José Antonio Páez (Arauca).

»Colombia-Venezuela: Paso de frontera Paraguachón (Guajira).

Si bien, Colombia tiene muchas similitudes en cuanto al idioma, cultura y tradiciones con Venezuela, no es fácil para los venezolanos llegar a un país distinto donde deben adaptarse y salir en busca de oportunidades educativas, laborales y de salud. Es allí, donde las bibliotecas públicas, en este caso, de la ciudad de Medellín, deben entrar a jugar un papel preponderante en la adaptación y disposición de sus instalaciones, recursos y servicios para esos nuevos usuarios que van llegando, como lo son las personas inmigrantes, apelando a la función social de la biblioteca y su multiculturalidad.

Es importante aclarar el concepto de migrante e inmigrante para efecto de este trabajo. La Organización Internacional para la Migraciones (OIM) define a un migrante como

cualquier persona que se desplaza o se ha desplazado a través de una frontera internacional o dentro de un país, fuera de su lugar habitual de residencia independientemente de: 1) su situación jurídica; 2) el carácter voluntario o involuntario del desplazamiento; 3) las causas del desplazamiento; 0 4) la duración de su estancia. (OIM, 2018).

Es decir, migrante es aquel que sale de su país, ciudad o pueblo para establecerse en otro, e inmigrante es quien llega a un país para establecerse en él.

\section{Ciudadanos venezolanos en Colombia}

En el informe Radiografía de venezolanos en Colombia, según Christian Krüger Sarmiento, Director General de Migración Colombia,

Para hablar de migración entre Colombia y Venezuela es necesario tener en cuenta tres aspectos fundamentales. El primero de ellos, tiene que ver con la historia, pues al ser países vecinos el movimiento de personas entre las dos naciones ha sido permanente. Debemos recordar, que décadas atrás, éramos nosotros, los colombianos, quienes viajábamos a buscar oportunidades al vecino país. El segundo aspecto, tiene que ver con el crecimiento de los flujos migratorios de los 


\begin{abstract}
últimos años, que mientras con el resto de países es del 11\%, con Venezuela este crecimiento alcanza el $15 \%$. Y un tercer aspecto, tiene que ver con la dinámica que se presenta actualmente, donde observamos una migración pendular, es decir que se mueve entre los dos países en zona de frontera regularmente. Una migración regular, es aquella que ingresa por puntos habilitados sellando su pasaporte y, en algunos casos, tiene una vocación de residencia. Y una última migración, la cual es de tránsito y utiliza nuestro país como puente para llegar a terceros países" (Migración Colombia, 2017).
\end{abstract}

Migración Colombia, estima que en diciembre de 2017 ingresaron al país 109.649 venezolanos; cifras oficiales apuntan que finalizando el 2017, en el país se encontraban más de 550.000 venezolanos. Esto significó un aumento cercano al $62 \%$ respecto al mismo informe presentado a mediados del año 2016 (Migración Colombia, 2018a).

Hasta el 31 de diciembre de 2017, se tenían registrados más de 1.300 .000 ciudadanos venezolanos como usuarios de la Tarjeta de Movilidad Fronteriza (TMF).

Una de las estrategias que el gobierno nacional utilizó para regular la situación migratoria de aquellos ciudadanos venezolanos que antes del 28 de julio de 2017 habían ingresado de forma legal al país, consistió en la implementación del Permiso Especial de Permanencia (PEP), el cual es un documento gratuito expedido por el gobierno colombiano, a través de Migración Colombia tiene una vigencia de 2 años a partir de la fecha de su expedición y le permite a su portador regularizar su situación migratoria en el país, acceder al sistema bancario, trabajar, estudiar e incluso, cotizar en el sistema de salud y pensiones, así como también, desarrollar cualquier tipo de actividad legal dentro del territorio nacional, siempre que cumpla con los demás requisitos establecidos en el ordenamiento jurídico colombiano (Migración Colombia, 2018b).

Con relación a la ciudad de Medellín, los datos de la oficina de Migración Colombia, estiman que Medellín es la segunda ciudad del país donde más están llegando originarios de Venezuela en busca de refugio. Hasta diciembre de 2017, cerca de 69.000 ciudadanos venezolanos regularizaron su situación migratoria a través del PEP, de los cuales el $9 \%$ reside en Medellín (Migración Colombia, 2018).

\title{
3. La ciudad de Medellín
}

Medellín es la segunda ciudad en importancia en Colombia, y capital del Departamento de Antioquia; su temperatura promedio es de $24^{\circ} \mathrm{C}$ y está ubicada a 1.475 metros sobre el nivel del mar; cuenta con una extensión de 105 kilómetros cuadrados de suelo urbano, 270 de suelo rural y 5,2 de suelo para expansión. La ciudad está dividida en 6 zonas y 16 comunas, situada en el centro del Valle de Aburrá, en la Cordillera Central, y está atravesada por el río Medellín.

Según proyecciones del Departamento Administrativo Nacional de Estadística (DANE), Medellín cuenta con una población de 2.464.322 habitantes, lo que la hace la segunda ciudad más poblada de Colombia. Económicamente, Medellín es una ciudad que sobresale como uno de los principales centros financieros, industriales, comerciales y de servicios de Colombia, primordialmente en los sectores textil, confecciones, metalmecánico, eléctrico y electrónico, telecomunicaciones, automotriz, alimentos y salud (Medellín cómo vamos, 2018).

Los factores anteriores, sumado a los índices constantes de crecimiento; convierte la ciudad de Medellín en un lugar muy atractivo para locales, nacionales y extranjeros en materia de turismo e inversión, además, cuenta con una excelente red de 
transporte, movilidad y servicios públicos, siendo un ejemplo a seguir en Colombia por su urbanismo, calidad de vida y ciudadanía, lo que la ha hecho acreedora del premio como la ciudad más innovadora del mundo en el año 2013 y en el 2016 fue galardonada con el premio más importante de urbanismo, el Lee Kuan Yew World City Prize, considerado el "Nobel de las ciudades".

Es por ello que Medellín se ha convertido, actualmente, en una ciudad predilecta para la llegada de ciudadanos venezolanos que desean radicarse de manera permanente en Colombia, dado que les brinda más y mejores oportunidades laborales y académicas que su país de origen.

\section{Las bibliotecas públicas de Medellín}

Las bibliotecas públicas son lugares llamativos debido a la gratuidad de sus servicios e instalaciones, por esta razón, muchas personas inmigrantes acuden a ellas en busca de información, cultura, ocio y recreación.

"La biblioteca pública presta sus servicios sobre la base de igualdad de acceso de todas las personas, independientemente de su edad, raza, sexo, religión, nacionalidad, idioma o condición social. Ha de contar además con servicios específicos para quienes por una u otra razón no puedan valerse de los servicios y materiales ordinarios, por ejemplo, minorías lingüísticas, deficientes físicos y mentales, enfermos o reclusos" (UNESCO, 1994).

La biblioteca, al ser un espacio de libre acceso, acoge a toda clase de usuarios, por tal motivo, es concebida como un espacio dinámico que propicia el encuentro, la reunión e interacción entre las personas que acuden a ella. Es precisamente esa diversidad la que le da un sentido democrático y de cohesión social.

Medellín cuenta con El Sistema de Bibliotecas Públicas (SBPM), que tiene entre sus propósitos garantizar el libre acceso a la información, a la lectura, a la escritura, al conocimiento, al pensamiento y al disfrute de la cultura, de todos los ciudadanos.

Está conformado por 35 unidades de información, ubicadas en puntos estratégicos de 16 comunas y 5 corregimientos de la ciudad. Las bibliotecas, en sus diferentes tipologías, no solo son espacios para la consulta de información en diferentes formatos, sino que también, son lugares acogedores e incluyentes, que ofrecen información pertinente, formación para el desarrollo humano integral y oportunidades para el encuentro y la construcción colectiva. Lo que las convierte en centros de desarrollo comunitario y local (Sistema de Bibliotecas Públicas de Medellín, 2018).

El Sistema de Bibliotecas se constituye de la siguiente manera:

a) Nueve Parques Biblioteca: entendidos como centros de desarrollo cultural, que trasciende el concepto tradicional de la Biblioteca, debido a su infraestructura y oferta de servicios que permiten fortalecer el vínculo con la comunidad.

b) Doce Bibliotecas de Proximidad: recoge las bibliotecas públicas de pequeño y mediano formato, hoy reconocidas como referente barrial o corregimental.

c) Siete Centros de Documentación: entendidos como unidades de información, que aportan en el eslabón de la cadena del conocimiento, supliendo necesidades específicas y especializadas, como la planeación urbanística, memoria, medio ambiente y primera infancia. 
d) La Biblioteca Pública Piloto para América Latina y sus cuatro filiales: ente descentralizado del Municipio de Medellín, cumple funciones de Biblioteca Patrimonial para la ciudad, teniendo en su custodia gran parte del Archivo fotográfico de la ciudad.

Considerando que Colombia carece de una política para atender a inmigrantes, debido a que nunca antes se había visto enfrentado una inmigración de tal magnitud, dicha inexperiencia por parte del gobierno colombiano se ha evidenciado en las medidas coyunturales que se han tomado sobre la marcha. Es por ello que la biblioteca pública debe contribuir al desarrollo de la comunidad a la que atiende, buscando estrategias que le permitan incluir e integrar a personas inmigrantes que van llegando a ella, por el atractivo de sus diferentes espacios y servicios.

Según las directrices de la IFLA/UNESCO para el desarrollo del servicio de bibliotecas públicas, la biblioteca debe esforzarse en poner a disposición sus diferentes recursos, para lo cual deberá organizar "Servicios a inmigrantes y nuevos ciudadanos para ayudarles a encontrar su camino en una sociedad diferente y darles acceso a los medios de comunicación de su cultura de origen"(IFLA, 2001).

En el año 2008, La Federación Internacional de Asociaciones de Bibliotecarios y Bibliotecas (IFLA) por sus siglas en inglés, amplió estas directrices, otorgando una visión más amplia acerca de la función multicultural de la biblioteca, dejando en claro que se debe prestar especial atención a los grupos culturalmente diversos, por tal razón,

\begin{abstract}
Los servicios bibliotecarios y de información en un contexto de diversidad cultural y lingüística incluyen tanto la oferta de servicios a todo tipo de usuarios de la biblioteca como la oferta de servicios bibliotecarios dirigidos especialmente a grupos culturales y lingüísticos tradicionalmente más desatendidos. Se debería prestar especial atención a grupos que a menudo sufren marginación en las sociedades con diversidad cultural: minorías, refugiados y solicitantes de asilo, personas con permisos de residencia temporales, trabajadores inmigrantes y comunidades indígenas (IFLA, 2009).
\end{abstract}

\title{
5. Servicios bibliotecarios más utilizados por inmigrantes venezolanos
}

En Medellín, las bibliotecas públicas se han caracterizado por conectar territorios, intercambiar conocimientos, brindar buenos servicios bibliotecarios y ser espacios incluyentes, dinámicos y de conversión ciudadana.

Para el ejercicio de este trabajo, se solicita de manera formal a las coordinaciones de las diferentes bibliotecas de la ciudad de Medellín, principalmente, a los 9 parques biblioteca y bibliotecas de proximidad; información acerca de programas, servicios, actividades, experiencias significativas o estadísticas de utilización de servicios por parte de ciudadanos venezolanos en sus unidades de información, aunque, en su mayoría han visibilizado usuarios ocasionales, son solo algunas bibliotecas las que han identificado un aumento considerable en la asistencia y utilización de dichos espacios y servicios.

Es importante acotar, que si bien los ciudadanos venezolanos, como cualquier otro ciudadano, independientemente de su nacionalidad, pueden hacer uso de todos los espacios, servicios, programas y recursos que ofrece día a día la biblioteca pública, se busca identificar cuáles son esos servicios a los que más acceden y si es el caso, qué actividades o programas especiales ofrece la biblioteca para ellos. 
El Parque Biblioteca Belén (PBB) se encuentra en uno de los sectores más poblados de Medellín, ubicada en la comuna 16 de la zona suroccidental de la ciudad. Es un espacio de la Alcaldía de Medellín en cooperación con la caja de compensación familiar Comfenalco Antioquia. Constituye un eje integrador de los 23 barrios que forman la comuna, con un gran valor paisajístico. Entre sus proyectos y servicios se encuentran: Escuela de Música, Centro Público de Empleo, Centros de Desarrollo Empresarial Zonal (Cedazo), Centro de Documentación Buen Comienzo y Centro de Servicios de Comfenalco (Red de Bibliotecas, 2018).

El PBB al estar inmerso en la segunda comuna más grande de la ciudad, con una mayor población e infraestructura, al igual que su cercanía al centro de Medellín, la convierte en una biblioteca dinámica e integradora, siendo tal vez, el Parque Biblioteca más concurrido de la ciudad.

Las estadísticas del PBB han reflejado un aumento significativo de ciudadanos venezolanos en la utilización de los servicios bibliotecarios, tales como: consulta en sala general, talleres de sala infantil, consulta en sala de internet y demás espacios de la biblioteca. Esto se debe al factor geográfico anteriormente mencionado, sumado a la cercanía del Parque Biblioteca con el consulado de la República Bolivariana de Venezuela y la sede de Migración Colombia en Medellín, ubicados en diferentes barrios de Belén.

Sala Mi Barrio es un espacio de ciudadanía e información local, que hace parte de los servicios del Parque Biblioteca Belén. Entre las actividades que ofrece al público, se encuentran los talleres de creación y actualización de hojas de vida, donde los usuarios venezolanos asisten de manera recurrente, aunque desde hace algún tiempo, dicho servicio se ha redireccionado al Centro Público de Empleo que se halla dentro del PBB y el cual, ha venido cumpliendo la misma función.

Cabe destacar que en el 2017 se realizó una campaña solidaria que permitió recolectar ropa y víveres para la comunidad venezolana recién llegada. Dicha actividad se desarrolló en sinergia con la biblioteca y voluntarios venezolanos. Evidenciando el compromiso y responsabilidad que tiene el PBB con su labor, fortaleciendo el vínculo con la comunidad.

El servicio que se ofrece en Sala de Internet en el PBB, permite a los usuarios navegar libremente en la web durante 1 hora, con la posibilidad de ingresar varias veces al día (usos). La tabla a continuación, muestra la cantidad de usuarios y usos del servicio durante el primer cuatrimestre del año 2018:

\begin{tabular}{rcccc}
\cline { 2 - 4 } & Enero & Febrero & Marzo & Abril \\
\hline Usos & 524 & 958 & 606 & 1147 \\
Usuarios & 61 & 97 & 58 & 135 \\
\hline
\end{tabular}

Fuente: Estadística Sala de internet PBB

Teniendo en cuenta lo anterior, se puede afirmar que en promedio cada usuario venezolano en la sala de internet realiza aproximadamente 9,3 usos al mes.

En el año 2006 se inauguró el primer Parque Biblioteca Pbro. José Luis Arroyave Restrepo, ubicado en el sector de San Javier, comuna 13 de Medellín. Es un espacio de vida donde existen zonas verdes y espacios abiertos, entre sus servicios se encuentra Sala Mi Barrio, exposiciones, ludoteca, aulas de estudio, entre otros. 


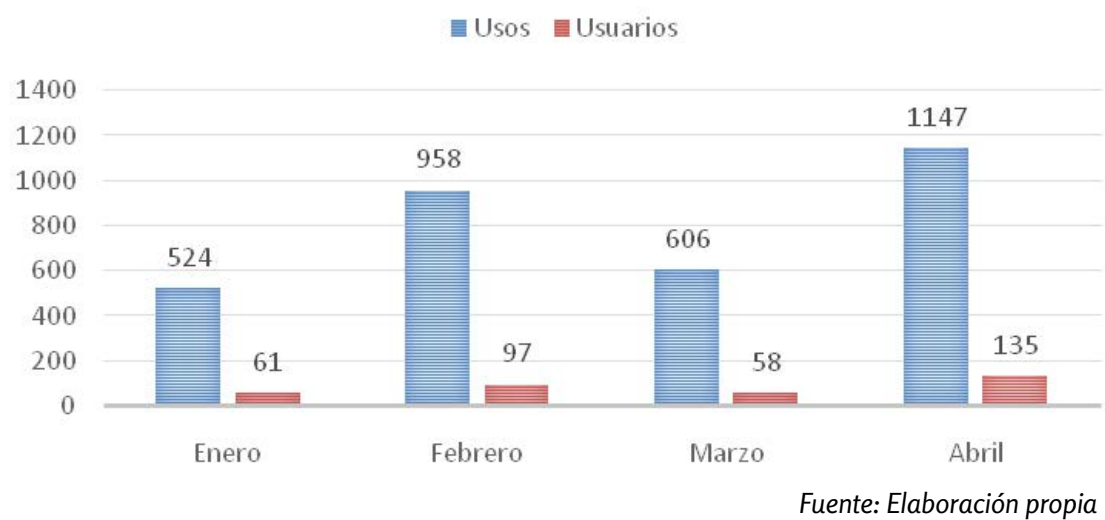

En este Parque Biblioteca se ha evidenciado una afluencia importante de venezolanos que utilizan los servicios bibliotecarios anteriormente mencionados, así como también, el uso de la sala virtual, consulta general en sala y los demás programas con los que cuenta la biblioteca.

Hasta marzo del presente año, 67 ciudadanos venezolanos, entre ellos 41 hombres y 26 mujeres han hecho uso de los servicios de sala virtual, representados en la Tabla 2.

\begin{tabular}{|l|c|}
\hline Grupo poblacional & Cantidad \\
\hline Población infantil & 1 \\
\hline Niños & 2 \\
\hline Adolescentes & 8 \\
\hline Jóvenes & 28 \\
\hline Adultos & 27 \\
\hline Adultos mayores & 1 \\
\hline
\end{tabular}

Fuente: Estadística Sala virtual, P.B. Pbro. José Luis Arroyave Restrepo-San Javier
Tabla 2. Segmentación de usuarios PB Prbo. José Luis Arroyave Restrepo-San Javier

En el Gráfico 2, se evidencia que la mayor cantidad de usuarios que utilizan los servicios de sala virtual son jóvenes y adultos, representando el $42 \%$ y $40,1 \%$ respectivamente del total de usuarios de nacionalidad venezolana.

Algo similar ocurre en el Parque Biblioteca León de Greiff situado en La Ladera, comuna 8, donde se tiene el registro de utilización de servicios, principalmente el centro de navegación en internet. Hay que resaltar que el Parque Biblioteca ha venido articulándose con organizaciones sociales de su comunidad que trabajan y tienen conocimiento del territorio, por ejemplo; la Fundación Golondrinas, quienes lideran procesos que permiten conocer el censo poblacional de ciudadanos venezolanos que se han establecido en su comuna, siendo un aproximado de 1200 venezolanos, según su director Jesús Antonio Marulanda. De modo que el aprovechamiento de esta información por parte de la biblioteca, se convierte en una oportunidad importante para la proyección de servicios que permitan atraer a estos nuevos usuarios. 
Gráfico 2. Segmentación de usuarios venezolanos $P B$ Prbo. José Luis Arroyave Restrepo

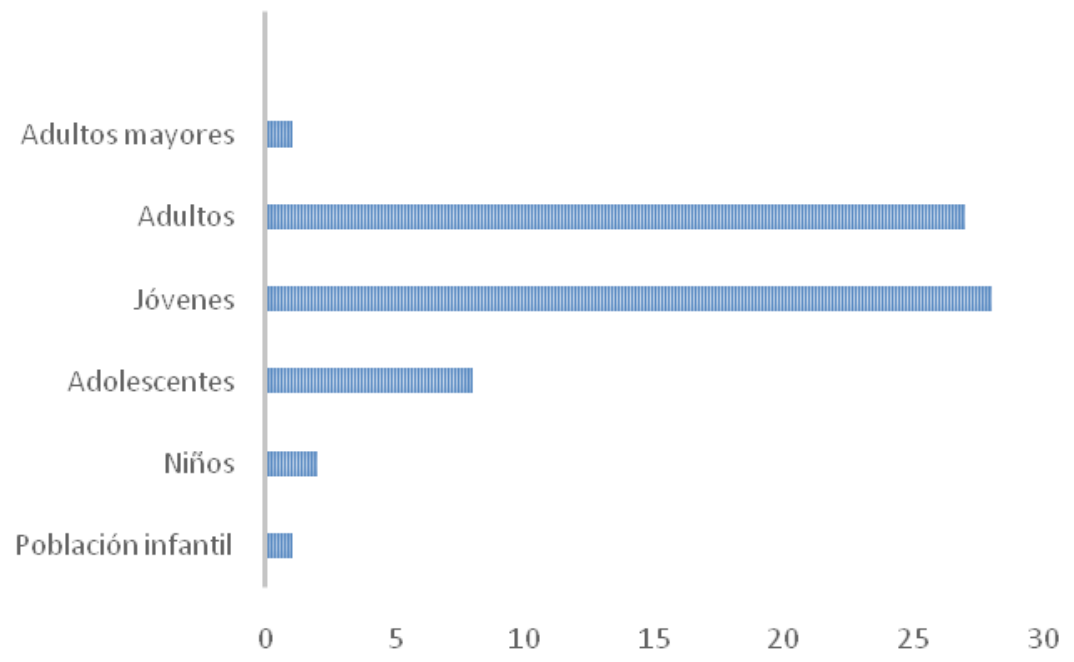

Fuente: Elaboración propia.

La Biblioteca Pública El Poblado con poco más de 1 año de fundación, está ubicada en la parte alta de la comuna 14 Poblado, dicha comuna es la más grande de Medellín y menos poblada en términos relativos, en la cual predomina un nivel socio económico alto. Allí asisten familias venezolanas que participan en diversas actividades programadas en la biblioteca como:

"Pasitos lectores, un espacio pensado para la estimulación en los pequeños entre los 3 meses y los 6 años a partir de los libros y la lectura.

"Abuelos cuenta cuentos.

"Club de lectura.

»Club de conversación en inglés.

Entre las experiencias significativas de la biblioteca, se encuentra la realización de tertulias en el año 2017, en la cual se trató el tema de inmigrantes, teniendo como invitada una participante venezolana que compartió con los asistentes su experiencia de vida en la ciudad.

Una de las bibliotecas ubicadas en el centro de la ciudad, al lado de la Casa de la Lectura Infantil, es la biblioteca pública Héctor González Mejía, que hace parte de la Red de Bibliotecas de la caja de compensación Comfenalco Antioquia.

Según la oferta del servicio de referencia, con respecto a la consulta de trámites de asuntos legales y migratorios por parte de usuarios venezolanos, la biblioteca con el ánimo de satisfacer la creciente demanda de información requerida, decidió publicar por medio del sitio web del Servicio de Información Local (Infolocal), la descripción y requisitos necesarios para acceder al Permiso Especial de Permanencia que expide Migración Colombia. De tal modo, que los usuarios puedan consultar de una manera fácil y directa la información por medio de la página, asimismo, hacer uso de la sala de cómputo con la cual dispone la biblioteca.

La Biblioteca EPM, ubicada en la Plaza Cisneros de Medellín, es una biblioteca especializada en ciencia, industria, tecnología y medio ambiente. Por su ubicación en pleno 
corazón de la ciudad, garantiza el acceso del público en general, siendo notorio en los últimos tiempos, la utilización de servicios por parte de ciudadanos venezolanos.

Los servicios que más utilizan son:

"Sala de audio y video: Proyección de películas cinematográficas.

"Sala de internet: acceso a computadores e internet.

» Punto Info: servicio de información local; allí es contante la asesoría a ciudadanos venezolanos en la creación de su hoja de vida, y orientación y manejo de la plataforma del Servicio Público de Empleo, también brinda información sobre sitios de interés como la oficina de Migración Colombia y Cancillería venezolana, entre otros.

Cabe aclarar que los dos primeros servicios, sala de audio y video y sala de internet, no se tuvieron que adaptar a la comunidad venezolana, sino que son utilizados con mayor frecuencia por dicha población.

El panorama anterior, muestra cómo en su función social y democrática, las bibliotecas han realizado esfuerzos por ofrecer un mejor servicio a esos nuevos usuarios procedentes de Venezuela, se ha demostrado cómo algunas bibliotecas, sobre todo las que han detectado una mayor asistencia de población venezolana, han tomado la iniciativa de recopilar información necesaria, por medio de estadísticas concretas, que les permita conocer la número exacto de usuarios y usos reales de los servicios, así como también, identificar cuáles son los servicios bibliotecarios más utilizados por los mismos. Por otro lado, el fortalecimiento y la articulación con los actores sociales de las comunidades en las que están inmersas las bibliotecas públicas.

La función multicultural de la biblioteca puesta en práctica, como lo explica Lozano Díaz

implicará en muchas ocasiones un cambio de procesos y normas que se tendrán que reelaborar y reinterpretar con mente abierta y tolerante para facilitar que toda persona recién llegada a nuestra comunidad tenga su lugar y su punto de apoyo en la biblioteca pública, independientemente de su función administrativa (Lozano Díaz, 2006: 39).

Si bien, la mayoría de las bibliotecas como la Héctor González Mejía de Comfenalco Antioquia, el Parque Biblioteca San Antonio de Prado, Biblioteca Manuel Mejía Vallejo-Guayabal, Biblioteca La Floresta, Parque Biblioteca Fernando Botero-San Cristóbal, Biblioteca El Poblado, entre otras que conforman el Sistema de Bibliotecas, evidencian un auge en la utilización de espacios y servicios por parte de usuarios venezolanos, se puede concluir que entre las unidades de información más concurridas, están los Parques Biblioteca de San Javier, León de Greiff en la Ladera y el Parque Biblioteca Belén, siendo esta última, la biblioteca que registra un número mayor de ciudadanos venezolanos que hacen usos de sus servicios.

\section{Algunas estrategias}

La biblioteca pública debe procurar la búsqueda de espacios y estrategias que permitan la inclusión e integración de las personas nuevas que llegan en busca de la utilización de sus servicios, como lo son las personas provenientes del hermano país de Venezuela. 
Algunas estrategias que pueden implementarse en la biblioteca pública son las siguientes:

»Promover una cultura de respeto, tolerancia y solidaridad hacia el inmigrante que visita la biblioteca.

"Hacer pública la guía de derechos y deberes para venezolanos en Colombia, realizada por el Grupo de Aplicación de Derecho Internacional en Colombia GADIC del Consultorio Jurídico - Universidad del Rosario, la cual contiene temas de interés, rutas de atención y servicios migratorios para que las personas recién llegadas puedan tener acceso a ella. (Grupo de Aplicación de Derecho Internacional en Colombia (GADIC), Consultorio Jurídico - Universidad del Rosario)

"Realizar formación de usuarios y recorridos bibliotecarios, que permita a los ciudadanos venezolanos familiarizarse con el funcionamiento de la biblioteca.

»Evaluar las necesidades generales de los inmigrantes a través de encuestas y entrevistas a grupos focales.

» Brindar información oficial y actualizada sobre los diferentes trámites, solicitudes de asilo y permisos de residencia para inmigrantes.

» Proveer información que permita la lectura del contexto colombiano, en el ámbito local, regional y nacional.

" Capacitar en temas de compromiso y ciudadanía, asimismo, en educación, salud, empleo, rutas de atención y servicios sociales para inmigrantes, como el asesoramiento en trámites de solicitudes de asilo, cédula de extranjería, permisos de residencia, convalidación de estudios realizados, entre otros.

"Propiciar un espacio para la convivencia, por medio de la pedagogía de los usuarios sobre el tema de migración, que facilite la desestigmatización de los mismos.

»Crear estrategias que permitan atraer a aquellos ciudadanos venezolanos que no hayan tenido acceso a las bibliotecas en su país.

" Incorporar de forma sistemática en la biblioteca, herramientas que permitan recopilar información estadística y/o indicadores sobre los usos y servicios por parte de usuarios venezolanos.

» Tener dentro de la colección, diversos materiales bibliográficos disponibles, por ejemplo; literatura venezolana, que permita seguir manteniendo el vínculo con su país de origen.

"Entablar redes de cooperación entre la biblioteca pública y los diferentes grupos de apoyo a inmigrantes, con el fin de conocer las diversas necesidades que permita ofrecer un mejor servicio.

» Brindar programas de alfabetización digital.

" Facilitar las tecnologías de la información que posibiliten la comunicación y el contacto con sus familiares y amigos.

" Realizar campañas de solidaridad de apoyo a inmigrantes venezolanos, ya sea por medio de la recolección de alimentos, vestuario o útiles escolares para quien lo necesite.

» Hacer uso del Servicio de Información Local (SIL) para la difusión de actividades culturales, residencias en alquiler, ofertas laborales, entre otros.

» Capacitar al personal bibliotecario para una adecuada mediación de las personas inmigrantes.

" Programar actividades de lectura, charlas, foros o tertulias que propicien un espacio de encuentro e intercambio cultural entre las dos naciones.

» Ofrecer el servicio de préstamo de material bibliográfico con el documento de residencia o permisos especiales.

» Realizar eventos de bibliotecas humanas, donde los usuarios además de encontrar libros tradicionales, hallen personas con historias que contar y dialogar sobre el tema de inmigración, comunidades venezolanas y la riqueza cultural y tradicional de los dos países. 


\section{Conclusiones}

Está claro que las bibliotecas públicas de la ciudad de Medellín, prestan sus servicios sobre la igualdad de derechos y acceso, sin importar su lugar de procedencia. La mayoría de bibliotecas no cuentan con información específica de la totalidad de venezolanos que acuden a ellas, por lo que se toma en cuenta, la observación por parte del personal bibliotecario y la estadística general de los extranjeros que la visitan y asisten a las diferentes actividades y programas que se llevan a cabo.

Por otro lado, las unidades de información que realizan un registro estadístico de sus usuarios, muestran un creciente uso de los servicios bibliotecarios por parte de inmigrantes venezolanos, sobre todo en la utilización de los espacios, servicios de información local y salas virtuales, teniendo en cuenta la inexistencia de una política unificada que facilite el préstamo de material bibliográfico, debido a que aún no hay claridad en cuanto a los permisos y regularización de la situación migratoria, que permita establecer qué tipo de documento se debe requerir para dicha solicitud, exceptuando aquellos ciudadanos venezolanos que poseen cédula de extranjería o doble nacionalidad con la cual pueden acceder al servicio de préstamo.

Si las bibliotecas trabajan mancomunadamente en la planeación de objetivos y políticas claras interbibliotecarias, ya sea a largo o corto plazo, que incluya el préstamo de material bibliográfico o la realización de programas y actividades, sería mucho más fácil prestar servicios que contribuyan tanto a la adaptación y generación de nuevas oportunidades, como al fortalecimiento de la identidad de los inmigrantes.

\section{Consideraciones finales}

Hay que entender que el análisis de la comunidad y el estudio de usuarios reales y/o potenciales, es un instrumento que facilita el reconocimiento e identificación sobre quiénes y cuáles son los usuarios y grupos que acuden a las unidades de información, también sobre el cómo y cuándo utilizan los servicios, lo cual ayuda a la toma de decisiones, optimizar, direccionar, crear nuevos servicios, recursos, programas y estrategias que proporcionen el mejoramiento y la calidad de vida de las personas.

Es importante reconocer la diversidad, saber que existen necesidades y expectativas diferentes, grupos de usuarios que pueden hacer uso de los servicios tradicionales bibliotecarios o necesitar la demanda de información especial. Es preciso insistir en dejar a un lado la "neutralidad" que caracteriza un sector de la biblioteca pública, "proporcionar servicios bibliotecarios en forma neutral significa solamente cumplir con un cierto modelo de igualdad que justifica la hegemonia pero niega la existencia de las diferencias. De alli que el verdadero servicio igualitario se tendria que buscar, tomando como base la atención de las diferencias" (Candame et al., 2007: 52).

El desarrollo de servicios bibliotecarios multiculturales, conlleva a unificar esfuerzos entre la biblioteca pública, el personal bibliotecario, la comunidad de usuarios, los gobiernos locales y diferentes organismos de apoyo a inmigrantes, que posibilite un estudio a fondo del contexto, características y necesidades de la población a la que sirve, adaptándose a la realidad del entorno que la rodea. Es recomendable que las bibliotecas y profesionales de la información, sean conscientes de la responsabilidad social que urge en el quehacer diario, así como también, estar al tanto de las pautas y directrices de la IFLA con respecto a los Servicios Bibliotecarios Multiculturales (Multicultural Communities: Guidelines for Library Services), no obstante, aunque no correspondan al contexto latinoamericano, pueden ser útil como referencia y guía (IFLA, 2009). 
La realidad actual sugiere que muchos ciudadanos del vecino país seguirán llegando a la ciudad en busca de nuevas oportunidades. Por consiguiente, las bibliotecas públicas y, sobre todo, aquellas que ya estén viviendo este fenómeno, deberán adaptarse a su entorno y buscar estrategias para fortalecer la función integradora e incluyente que las acomete, generar políticas claras y unificadas que permitan orientar servicios bibliotecarios para inmigrantes venezolanos.

La biblioteca pública está llamada, entonces, a ser partícipe de estas nuevas transformaciones sociales y culturales, como institución democrática y de desarrollo social, propiciar el respeto y la integración que conlleve a un proceso de interculturalidad ciudadana. 


\section{Q Referencias bibliográficas}

"Candame, Rita; Tatiana Carsen; Oscar Maya y Martín Vera. 2007. Responsabilidad y compromiso social de los profesionales de la información (bibliotecarios, documentalistas, archivistas): una visión latinoamericana. En Gimeno Perelló, Javier; Pedro López López y María Jesús Morillo Calero, coords. De volcanes llena: biblioteca y compromiso social. Gijón: Trea. p. 49-70.

» Grupo de Aplicación de Derecho Internacional en Colombia (GADIC). Consultorio Jurídico, Universidad del Rosario. 2017. Guía de derechos y deberes para venezolanos en Colombia. <http://www.urosario.edu.co/consultorio-juridico/Documentos/Cartilla-Derechos-y-Dereberes-Venezolanos-en-Colom.pdf> [Consulta: agosto 2018].

»IFLA. 2001. Directrices IFLA/UNESCO para el desarrollo del servicio de bibliotecas públicas. <https://www.ifla.org/files/assets/hq/publications/archive/the-publiclibrary-service/pgo1-s.pdf> [Consulta: agosto 2018].

"IFLA. 2009. Comunidades multiculturales: directrices para el servivio bibliotecario. $<$ https://www.ifla.org/files/assets/library-services-to-multicultural-populations/ publications/multicultural-communities-es.pdf> [Consulta: agosto 2018].

》Lozano-Díaz, Roser. 2006. La biblioteca pública del S. XXI: atendiendo clientes, movilizando personas. Gijón: Trea.

»Medellín cómo vamos. 2018. <https://www.medellincomovamos.org/la-ciudad-2/> [Consulta: agosto 2018].

»Migración Colombia. 2017. Radiografía de venezolanos en Colombia. <http://www. migracioncolombia.gov.co/venezuela/radiografia_web.pdf> [Consulta: agosto 2018].

" Migración Colombia. 2018a. Estadísticas flujos de viajeros con uso de pasaporte. <http://migracioncolombia.gov.co/index.php/es/component/content/ article?id=718> [Consulta: 27 febrero 2018].

» Migración Colombia. 2018b. Permiso Especial de Permanencia (PEP). <http:// www.migracioncolombia.gov.co/index.php/es/prensa/comunicados/comunicados-2018/febrero-2018/6456-a-partir-de-hoy-se-habilita-plataforma-de-inscripcion-para-segunda-fase-del-permiso-especial-de-permanencia-pep-para-venezolanos> [Consulta: agosto 2018].

» Migración Colombia. 2018c. Radiografía migratoria Colombia-Venezuela. <http:// www.migracioncolombia.gov.co/index.php/es/prensa/multimedia/6308-radiografia-de-venezolanos-en-colombia-31-12-2017> [Consulta: 18 enero 2018].

»OIM. 2018. Organización Internacional para las Migraciones. <https://www.iom. int/es/quien-es-un-migrante> [Consulta: 12 marzo 2018].

"Red de Bibliotecas. 2018. Parque Biblioteca Belén. <http://reddebibliotecas.org. co/bibliotecas/pb-belen $>$ [Consulta: 2 abril 2018].

"Sistema de Bibliotecas Públicas de Medellín. 2018. http://bibliotecasmedellin.gov. $\mathrm{co} / \mathrm{cms} /$ nuestras-bibliotecas/> [Consulta: 23 febrero 2018].

》UNESCO. 1994. Manifiesto de la UNESCO sobre la biblioteca pública 1994. <http://unesdoc.unesco.org/images/oo11/o01121/112122so.pdf> [Consulta: agosto 2018]. 
